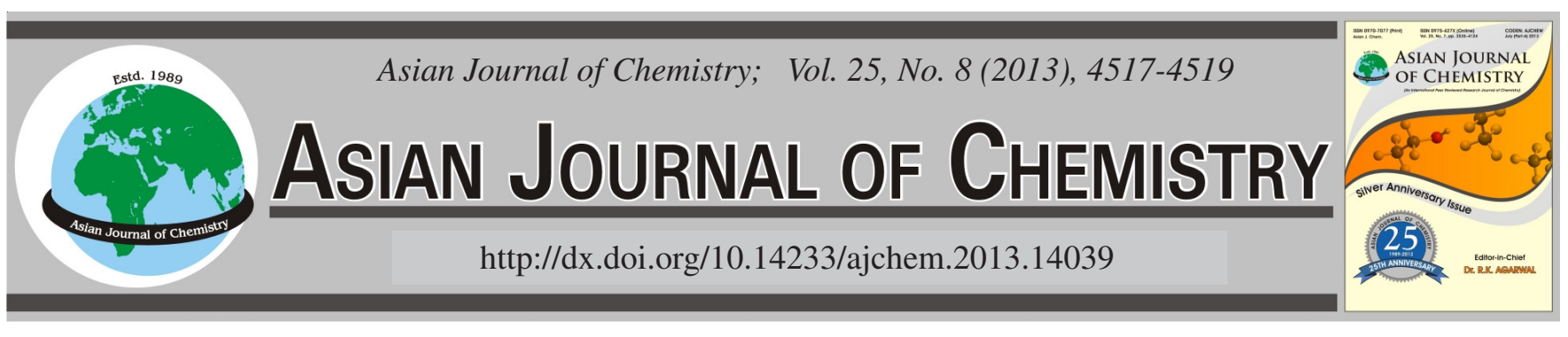

\title{
Electrochemical and Fluorescent Investigation of Inorganic-organic Hybrid from Butylrhodamine B and Keggin-Type Polyoxometalates
}

\author{
XiAOYing Zeng ${ }^{1}$, JuXing JiAng ${ }^{1}$, Haiyun Zhang ${ }^{2}$, Wenjing Lu ${ }^{2}$ and Dawei Pan ${ }^{2, *}$
}

${ }^{1}$ Research and Development Center, HongyunHonghe Tobacco (Group) Co. Ltd., Kunming 650202, P.R. China

${ }^{2}$ Key Laboratory of Coastal Zone Environmental Processes, Yantai Institute of Coastal Zone Research, Chinese Academy of Sciences, Shandong Provincial Key Laboratory of Coastal Zone Environmental Processes, YICCAS, Yantai Shandong 264003, P.R. China

*Corresponding author: Tel: +86 535 2109155; E-mail: dwpan@yic.ac.cn

\begin{abstract}
A new inorganic-organic hybrid composed of butylrhodamine B (BRB) and Keggin-type polyoxometalates $\left(\mathrm{PMo}_{12}\right)$ was introduced. The UV-visible and FTIR results indicate the formation of hybrid is caused by the interaction of butylrhodamine B and polyoxometallate. The $\mathrm{BRB}-\mathrm{PMo}_{12}$ hybrid modified electrode shows the good electrochemical properites and catalysis to the reduction of bromate. Additionally, at the same condition, the fluorescence intensity of $\mathrm{BRB}-\mathrm{PMo}_{12}$ hybrid is almost 1.3 times than that of butylrhodamine $\mathrm{B}$. The corresponding results have important implications for the design of multi-functional hybrid materials, which could be used widely in chemo/biosensing and catalysis.
\end{abstract}

Key Words: Butylrhodamine B, Polyoxometalates, Hybrid material, Electrochemistry, Fluorescence.

\section{INTRODUCTION}

The design and synthesis of inorganic-organic hybrid materials is of contemporary interest and becomes a major research theme in materials science ${ }^{1-3}$. The hybrid materials are expected to exhibit improved properties and functions not seen in pure inorganic or organic phases and combine distinct properties of organic and inorganic components within a single molecular composite. Organic materials offer structural flexibility, convenient processing and tunable electronic properties; while inorganic compounds provide the potential for high carrier mobility, a range of magnetic and dielectric properties as well as thermal and mechanical stability. Recent researches in this area have resulted in one-, two- and three-dimensional hybrid systems ${ }^{4-6}$.

As a large and rapidly growing class of compounds, polyoxometalates are inorganic metal-oxygen cluster compounds that are outstanding in their topological and electronic versatility and attracted attention in materials, biochemistry, medicine and catalysis ${ }^{7-9}$. Keggin-type polyoxometalates of molybdenum are particularly attractive due to their ability to accept various numbers of electrons giving rise to mixedvalency species. Lately, Keggin-type polyoxometalates were reported to stabilize platinum nanoparticles and carbon nanoparticles $^{10,11}$.

Rhodamine B and their derivatives dyes are a group of xanthene dyes and have high fluorescence quantum yield, which makes them very useful in applications like an active medium of dye lasers, fluorescence labeling and in bioanalytical chemistry ${ }^{12,13}$. Recently, the uses of rhodamine B compounds in synthesis of composite materials have been reported ${ }^{14-16}$.

In this paper, we have explored the preparation of inorganic-organic hybrid material by combining electronic polyoxometalates and fluorescent butylrhodamine B at the first time. The electrochemical and fluorescent characteristics of hybrid materials were discussed.

\section{EXPERIMENTAL}

Butylrhodamine B (BRB) and Keggin-type POM $\left(\mathrm{H}_{7} \mathrm{PMo}_{12} \mathrm{O}_{42}\right.$, abbreviated $\left.\mathrm{PMo}_{12}\right)$ were purchased from Chemical Reagent Company of Shanghai, China. All other reagents were of analytical grade and were used without further purification. Double-distilled water was used throughout.

Cyclic voltammetry measures were carried out in a conventional three-electrode cell controlled by CHI 660C Electrochemical Work Station (CH Instruments, Inc). Pyrolytic graphite (PG) electrode (surface area $0.06 \mathrm{~cm}^{2}$ ) was used as working electrode. A platinum foil was applied as the counter electrode and a saturated calomel electrode (SCE) served as reference electrode. All potential values given below refer to saturated calomel electrode. All experiments were performed at room temperature. The UV-VIS spectrum of BRB-PMo ${ }_{12}$ was recorded with a DU 800 spectrophotometer (Beckman, 
USA). The BRB-PMo ${ }_{12}$ adduct was pressed into a $\mathrm{KBr}$ pellet for FTIR investigation (WQF-410 spectrometer, Beijing, China). The fluorescence spectrum was obtained in F-4500 fluorescence spectrophotometer (Hitachi, Japan) at room temperature.

BRB-PMo ${ }_{12}$ hybrid was synthesized by addition of 0.05 $\mathrm{g} / \mathrm{mL}$ aqueous solution of butylrhodamine $\mathrm{B}$ dropwise to a solution containing $0.1 \mathrm{~g} / \mathrm{mL} \mathrm{PMo}_{12}$ under vigorous stirring. After complete addition, the mixture was stirred continuously for half an hour. The precipitates were centrifuged three times and washed each time with double distilled water to remove the excessive $\mathrm{PMo}_{12}$. Finally, dried overnight in a vacuum at room temperature and red-wine products can be obtained.

BRB-PMo ${ }_{12}$ hybrid was dispersed in $N, N$-dimethylformamide solution and sonicated for $5 \mathrm{~min}$ and result in $2 \mathrm{mg} / \mathrm{mL}$ homogeneous solution. Meanwhile, the pyrolytic graphite electrode (surface area $0.06 \mathrm{~cm}^{2}$ ) was polished with alumina, followed with being washed in double-distilled water and ethanol, respectively, then activated in $0.5 \mathrm{M} \mathrm{H}_{2} \mathrm{SO}_{4}$ solution. Then, $10 \mathrm{~mL}$ of BRB-PMo $\mathrm{Po}_{12}$ solution was added on the surface of pyrolytic graphite and dry in ambient.

\section{RESULTS AND DISCUSSION}

UV-visible spectroscopy is a useful and facile technique to evaluate the optical property of hybrid. Fig. 1 shows the $\mathrm{UV}$-visible absorption spectra of butylrhodamine $\mathrm{B}, \mathrm{PMo}_{12}$ and BRB-PMo ${ }_{12}$ hybrid. The $\mathrm{PMo}_{12}$ shows one strong characteristic peak at $211 \mathrm{~nm}$ (Fig. 1a), which can be ascribed to the O-Mo charge transfer transition. The butylrhodamine B exhibits one peak around $555 \mathrm{~nm}$ (Fig. 1b), which relates to the mononomeric form of butylrhodamine B. As shown in Fig. 1c, the hybrid presents two characteristic absorption peaks at $216 \mathrm{~nm}$ and $554 \mathrm{~nm}$, respectively, substantiating the optical characterization of butylrhodamine $\mathrm{B}$ and $\mathrm{PMo}_{12}$ in the hybrid do not alter each other.

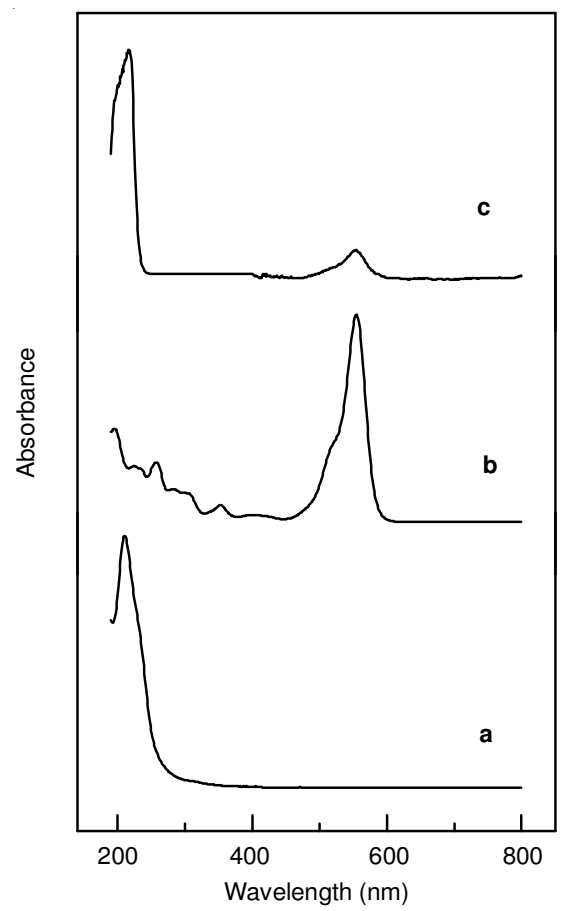

Fig. 1. UV-VIS spectra of (a) $\mathrm{PMo}_{12}$, (b) BRB and (c) BRB-PMo 12 solution
FTIR measurements were also carried out for $\mathrm{PMo}_{12}$, butylrhodamine $\mathrm{B}$ and $\mathrm{BRB}-\mathrm{PMo}_{12}$ hybrid. The corresponding results are shown in Fig. 2. The spectrum of $\mathrm{PMo}_{12}$ exhibits four obvious peaks at 1065, 968, 869 and $790 \mathrm{~cm}^{-1}$ (Fig. 2a). According to literatures ${ }^{10,17}$ peak at $1065 \mathrm{~cm}^{-1}$ is assigned to inner $\mathrm{P}-\mathrm{O}_{\mathrm{a}}-\mathrm{Mo}$ bond, peak at $790 \mathrm{~cm}^{-1}$ to $\mathrm{Mo}-\mathrm{O}_{\mathrm{d}}-\mathrm{Mo}$ bridges, peak at $869 \mathrm{~cm}^{-1}$ to $\mathrm{Mo}-\mathrm{O}_{\mathrm{b}}-\mathrm{Mo}$, while peak at $968 \mathrm{~cm}^{-1}$ is ascribed to external $\mathrm{Mo}=\mathrm{O}_{\mathrm{c}}$ bond. The formation of BRB$\mathrm{PMo}_{12}$ hybrid is evident from the FTIR spectrum because the unique bands of both $\mathrm{PMo}_{12}$ and butylrhodamine $\mathrm{B}$ can be observed on composite (Fig. 2c). Additionally, the spectrum of $\mathrm{PMo}_{12}$ is changed (from 1065 to 1052 , from 968 to 958 , from 869 to 880 , from 790 to 805 ) due to its adsorption on the butylrhodamine B dye.

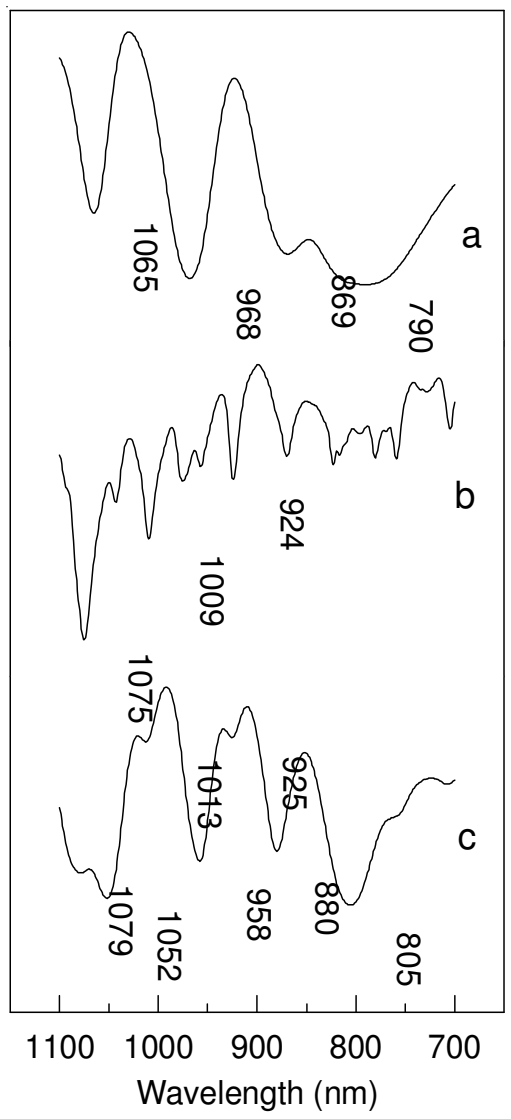

Fig. 2. FTIR spectra of (a) $\mathrm{PMo}_{12}$, (b) $\mathrm{BRB}$ and (c) $\mathrm{BRB}-\mathrm{PMo}_{12}$

The cyclic voltammogram of bare electrodes with BRB$\mathrm{PMo}_{12}$ modification in $0.5 \mathrm{M} \mathrm{H}_{2} \mathrm{SO}_{4}$ aqueous solutions is presented in Fig. 3. At BRB-PMo $\mathrm{PM}_{12}$ modified electrode, three reversible redox peaks can be observed clearly. The formal potential $\mathrm{E}_{1 / 2}\left(\mathrm{E}_{1 / 2}=\mathrm{E}_{\mathrm{Pa}} / 2+\mathrm{E}_{\mathrm{Pc}} / 2\right)$ for three peaks are +0.28 , $+0.18,-0.09 \mathrm{~V}$, respectively. The shape of the curves and the peak potentials are in accord with other work ${ }^{10}$. These results show that $\mathrm{PMo}_{12}$ in the hybrid still keeps its electrochemical properties. The electrocatalytical reduction of $\mathrm{BrO}_{3}^{-}$in $0.5 \mathrm{M}$ $\mathrm{H}_{2} \mathrm{SO}_{4}$ solution at BRB-PMo ${ }_{12}$ hybrid modified electrode was investigated preliminarily. It can be observed obviously that the reduction response currents decrease with the addition of the $\mathrm{BrO}_{3}^{-}$, which imply multi-reduction forms of $\mathrm{PMo}_{12}$ anion have electrocatalytic ability to the reduction of $\mathrm{BrO}_{3}^{-}$. 


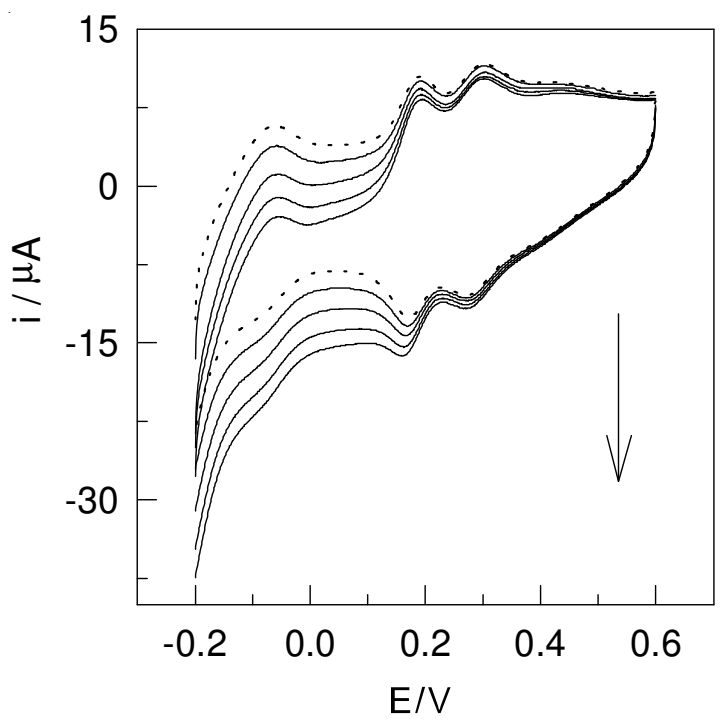

Fig. 3. Cyclic voltammograms of $\mathrm{PG} / \mathrm{BRB}-\mathrm{PMo}_{12}$ electrode in a $0.5 \mathrm{M}$ $\mathrm{H}_{2} \mathrm{SO}_{4}$ solution without (dotted line) and with 1, 2, 3, $4 \mathrm{mM}$ (from inside to outside, solid line) $\mathrm{BrO}_{3}$

The fluorescence excitation and emission spectra of butylrhodamine $\mathrm{B}$ and $\mathrm{BRB}-\mathrm{PMo}_{12}$ hybrid in DMF solution were shown in Fig. 4. It can be seen that butylrhodamine B solution shows apparent spectral characteristics with excitation maximum at $474 \mathrm{~nm}$ and emission maximum at $605 \mathrm{~nm}$. However, the wider distance between excitation and emission maximum and the lager fluorescence intensity can be observed at $\mathrm{BRB}-\mathrm{PMo}_{12}$ hybrid in the same experiment condition. The excitation maximum and emission maximum are at $496 \mathrm{~nm}$ and $599 \mathrm{~nm}$, respectively. The fluorescence intensity of BRB$\mathrm{PMo}_{12}$ hybrid is almost 1.3 times than that of butylrhodamine $\mathrm{B}$. These imply that the existence of $\mathrm{PMo}_{12}$ in hybrid does not change the original fluorescence property of butylrhodamine $\mathrm{B}$ but improve the fluorescence intensity because of the unique structure of $\mathrm{PMo}_{12}$.

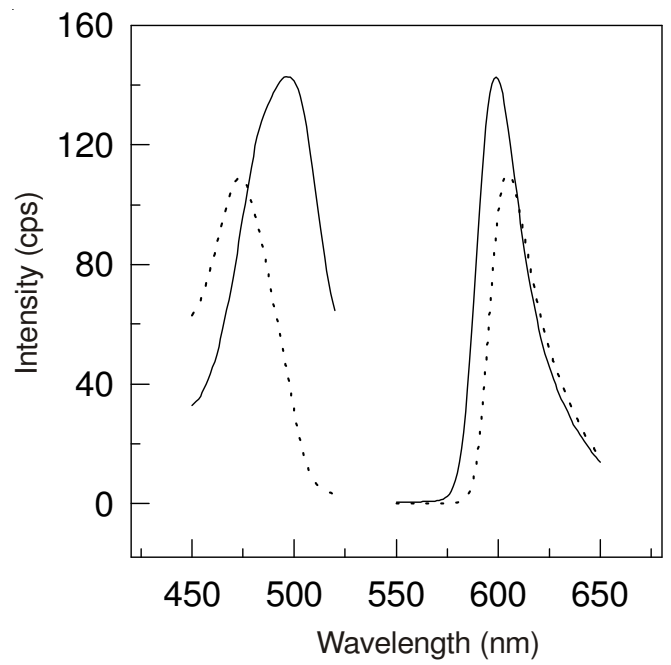

Fig. 4. Fluorescence spectrum of BRB solution (dotted line) and BRB$\mathrm{PMo}_{12}$ solution (solid line) in the same condition (PMT voltage $400 \mathrm{~V}$, Ex slit $5.0 \mathrm{~nm}$, Em slit $5.0 \mathrm{~nm}$ )

\section{Conclusion}

A new $\mathrm{BRB}-\mathrm{PMo}_{12}$ hybrid material was prepared here in a convenient and effective method. The resulting hybrid material can be used in chemically modified electrode for amperometric detection of bromate. More importantly, it has higher fluorescence intensity than pure organic phases. These results provide a useful avenue for preparing novel multifunctional hybrid materials, which can be used in catalysis, biosensing, magnetic and optical fields.

\section{ACKNOWLEDGEMENTS}

This work was financially supported by the National Natural Science Foundation of China (21007087), the Chinese Academy of Sciences (KZCX2-YW-JS208), the Natural Science Foundation of Shandong Province (BS2010HZ030), the Taishan Scholar Program of Shandong Province and the Youth Innovation Promotion Association of CAS.

\section{REFERENCES}

1. P.M. Forster, P.M. Thomas and A.K. Cheetham, Chem. Mater, 14, 17 (2004).

2. C. Cheng, S. Fu, C. Yang, W. Chen, K. Lin, G. Lee and Y. Wang, Angew. Chem. Int. Ed., 42, 1937 (2003).

3. S.S. Gu, S.S. Wang, Y.D. Yu and J.Y. Guo, Asian J. Chem., 23, 1241 (2011).

4. G.W. Hsieh, J.J. Wang, K. Ogata, J. Robertson, S. Hofmann and W.I. Milne, J. Phys. Chem. C, 116, 7118 (2012).

5. M.R.S.A. Janjua, M. Amin, M. Ali, B. Bashir, M.U. Khan, M.A. Iqbal, W. Guan, L.K. Yan and Z.M. Su, Eur. Inorg. Chem., 4, 705 (2012).

6. M.B. Gholivand and A. Azadbakht, Electrochim. Acta, 56, 10044 (2011).

7. R. Fazaeli, S. Tangestaninejad and H. Aliyan, Asian J. Chem., 22, 877 (2010).

8. P.J. Kulesza, B. Karwowska, B. Grzybowska and A. Wieckowski, Electrochim. Acta, 44, 1295 (1998).

9. L.H. Jin, Y.X. Fang, P. Hu, Y.L. Zhai, E.K. Wang and S.J. Dong, Chem. Commun., 48, 2101 (2012).

10. P.J. Kulesza, M. Chojak, K. Karnicka, K. Miecznikowski, B. Palys and A. Lewera, Chem. Mater., 16, 4128 (2004).

11. P. Garrigue, M. Delville, C. Labrugre, E. Cloutet, P.J. Kulesza, J.P. Morand and A. Kuhn, Chem. Mater., 16, 2984 (2004).

12. A.S. Ethiraj, N. Hebalkar, S. Kharrazi, J. Urban, S.R. Sainkar and S.K. Kulkarni, J. Lumin., 114, 15 (2005).

13. H. Chen, M. Zhou, X.Y. Jin, Y.M. Song, Z.Y. Zhang and Y.J. Ma, Anal. Chim. Acta, 478, 31 (2003).

14. F. Yang, Y. Chu, L. Huo, Y. Yang, Y. Liu and J. Liu, J. Solid State Chem., 179, 457 (2006).

15. Z. Klika, H. Weissmannova, P. Capkova and M. Pospisil, J. Colloid Interf. Sci., 275, 243 (2004).

16. Y. Shao, L. Wang, J. Zhang and M. Anpo, J. Photochem. Photobiol. A, 180, 59 (2006).

17. M. Fournier, C. Deltchef and L.P. Kazansky, Chem. Phys. Lett., 223, 297 (1994). 